\title{
Scenedesmus obliquus in poultry wastewater bioremediation
}

\author{
Ana Cristina Oliveira, Ana Barata, Ana P. Batista (i) and Luísa Gouveia \\ LNEG, National Laboratory of Energy and Geology I.P./Bioenergy Unit, Lisbon, Portugal
}

ABSTRACT

Wastewater biological treatment with microalgae can be an effective technology, removing nutrients and other contaminants while reducing chemical oxygen demand. This can be particularly interesting for the meat producing industry which produces large volumes of wastewater from the slaughtering of animals and cleaning of their facilities. The main purpose of this research was the treatment of poultry wastewater using Scenedesmus obliquus in an economical and environmentally sustainable way. Two wastewaters were collected from a Portuguese poultry slaughterhouse (poultry raw - PR and poultry flocculated - PF) and the bioremediation was evaluated. The performance of microalga biomass growth and biochemical composition were assessed for two illumination sources (fluorescent vs LEDs). S. obliquus achieved positive results when grown in highly contaminated agro-industrial wastewater from the poultry industry, independently of the light source. The wastewater bioremediation revealed results higher than $97 \%$ for both ammonium and phosphate removal efficiency, for a cultivation time of 13 days. The saponifiable matter obtained from the biomass of the microalga cultures was, on average, $11 \%$ and $27 \%\left(\mathrm{~m} / \mathrm{m}_{\text {alga }}\right)$ with PR and PF wastewater, respectively. In opposition, higher sugar content was obtained from microalgae biomass grown in PR wastewater (average $34 \% \mathrm{~m} / \mathrm{m}_{\text {alga }}$ ) in comparison to PF wastewater (average $23 \% \mathrm{~m} / \mathrm{m}_{\text {alga }}$ ), independently of the illumination source. Therefore, biomass obtained with PR wastewater will be more appropriate as a raw material for bioethanol/biohydrogen production (higher sugar content) while biomass produced in PF wastewater will have a similar potential as feedstock for both biodiesel and bioethanol/biohydrogen production (similar lipid and sugar content).

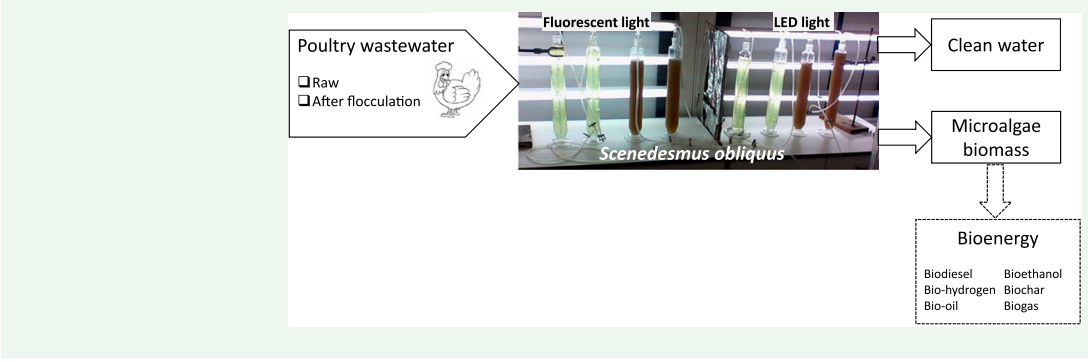

ARTICLE HISTORY Received 20 February 2018 Accepted 3 June 2018

\section{KEYWORDS}

Wastewater; microalga; Scenedesmus obliquus; poultry; bioremediation

\section{Introduction}

Global food production is estimated to be responsible for between $20 \%$ and $50 \%$ of anthropogenic environmental impacts [1] with the livestock sector accounting for more than the direct emissions from the transport sector [2].

While meat consumption is projected to increase by $70 \%$ by 2050 , chicken meat has become one of the most extensively consumed food products in the world [3]. Portugal ranks ninth in chicken production among European Union countries and broiler chicken represents $75 \%$ of the total meat consumption in this country [4]. Nevertheless, chicken has been described as the most environmentally friendly meat in several Life Cycle Assessment (LCA) studies (e.g. [1]). González-García et al. [4] studied a Portuguese broiler chicken production unit, namely the environmental impacts from the processes involved in a slaughterhouse (e.g. transport, packaging and waste treatment), with direct water emissions (e.g. $\mathrm{P}, \mathrm{COD}$ ) playing a significant impact in eutrophication potential. In fact, the meat processing sector produces large volumes of wastewaters due to the slaughtering of animals and cleaning of the slaughterhouse facilities and meat processing plants. This sector uses $24 \%$ of the total freshwater consumed by the food and beverage industry and up to $29 \%$ of that consumed by the agricultural sector worldwide [5]. In poultry processing, water is used primarily for feather removal (scalding), bird washing (before and after

CONTACT Ana Cristina Oliveira cristina.oliveira@Ineg.pt @ LNEG, National Laboratory of Energy and Geology I.P./Bioenergy Unit, Estrada do Paço do Lumiar 22, 1649-038 Lisbon, Portugal

(c) 2018 Informa UK Limited, trading as Taylor \& Francis Group 